\title{
Quantification of portal-bridging fibrosis area more accurately reflects fibrosis stage and liver stiffness than whole fibrosis or perisinusoidal fibrosis areas in chronic hepatitis C
}

Jérémy Sandrini ${ }^{1}$, Jérôme Boursier ${ }^{2,3}$, Julien Chaigneau ${ }^{2}$, Nathalie Sturm ${ }^{4}$, Jean-Pierre Zarski ${ }^{5,6}$, Brigitte Le Bail' ${ }^{7}$, Victor de Ledinghen ${ }^{8,9}$, Paul Calès ${ }^{2,3}$ and Marie-Christine Rousselet ${ }^{1,2}$

${ }^{1}$ Cellular and Tissue Pathology Department, University Hospital, CHU, Angers, France; ${ }^{2}$ HIFIH Laboratory, UPRES 3859, SFR 4208, LUNAM University, Angers, France; ${ }^{3}$ Liver-Gastroenterology Department, University Hospital, CHU, Angers, France; ${ }^{4}$ Pathology Department, University Hospital, Grenoble, France; ${ }^{5}$ Liver-Gastroenterology Department, University Hospital, Grenoble, France; ${ }^{6}$ INSERM/UJF U823, IAPC, IAB, Grenoble, France; ${ }^{7}$ Pathology Department, University Hospital, Bordeaux, France; ${ }^{8}$ LiverGastroenterology Department, Haut-Lévêque University Hospital, Pessac, France and ${ }^{9}$ INSERM U1053, Bordeaux Segalen University, Bordeaux, France

Morphometry provides an objective evaluation of fibrosis in liver diseases. We developed an image analysis algorithm using automated thresholding and segmentation to separately quantify the areas and the fractal dimensions of portal-bridging fibrosis and perisinusoidal fibrosis in chronic hepatitis $\mathrm{C}$ liver biopsies. We studied 427 digitized liver biopsies and compared the automated measures of the different fibrosis compartments with (1) the Metavir F (fibrosis) and A (activity) histological scores, (2) the digitally assessed area of steatosis, and (3) the liver stiffness measured by elastography (Fibroscan). The perisinusoidal fibrosis area was higher than that of portal fibrosis in stages $\leq$ F2; it reached its highest value in F2 stage and stabilized thereafter. The F3 stage was characterized by equal proportions of portal-bridging and perisinusoidal fibrosis, whereas portal-bridging area was predominant in cirrhosis. Measurement of portal-bridging fibrosis showed highly significantly different values between contiguous $F$ stages; the ratio of portal-bridging fibrosis/ perisinusoidal fibrosis displayed less overlap between Metavir stages than did the whole fibrosis area values. Fractal dimension showed that portal-bridging fibrosis tended to display a homogeneous surface-like spatial organization, whereas perisinusoidal fibrosis appeared more heterogeneous according to stage and curvilinear. The portal-bridging fibrosis area was low in cases with low Metavir activity and little steatosis, and became predominant with increasing activity and steatosis. Using stepwise multiple linear regression analysis, the liver stiffness was independently correlated to the portal-bridging fibrosis area (first step, $P<0.001$ ), the steatosis area (second step, $P<0.001$ ), and the Metavir A grade (third step, $P=0.001$ ), but not to the perisinusoidal fibrosis area. Automated quantification in a large cohort of chronic hepatitis $C$ showed that perisinusoidal fibrosis progressively grew in early fibrosis stages but did not increase in septal or cirrhotic stages and that the portal-bridging fibrosis area appeared as a more accurate tool to assess fibrosis progression than the whole fibrosis area.

Modern Pathology (2014) 27, 1035-1045; doi:10.1038/modpathol.2013.225; published online 3 January 2014

Keywords: automated segmentation; Fibroscan; image analysis; liver biopsy; morphometry

Correspondence: Professor M-C Rousselet, MD, Département de Pathologie Cellulaire et Tissulaire, CHU Angers, 49933 Angers, France.

E-mail: mcrousselet@chu-angers.fr

Received 30 June 2013; accepted 13 October 2013; published online 3 January 2014
Hepatitis C virus (HCV) infection is a worldwide disease that chronically affects $\sim 180$ million individuals. ${ }^{1}$ Monitoring of chronic HCV infection requires an evaluation of fibrosis, liver architectural modifications, and, less importantly, the extent of 
necroinflammation. This can be achieved by classic histopathologic examination of a liver biopsy specimen and the use of numerical scoring systems, such as the Metavir or Ishak scores. ${ }^{2,3}$ Noninvasive surrogates of histopathology, such as fibrosis blood tests and transient elastography, are nowadays used in daily clinical practice, mainly to detect clinically significant fibrosis and cirrhosis. ${ }^{4}$ The objective quantification of collagen deposition in liver biopsy material by different image analysis systems, ${ }^{5-7}$ although not routinely performed, gives a continuous measure of fibrosis, useful for statistical analyses, and reduces intra- and inter-observer variability. Moreover, such an objective quantification allows for valuable correlations with histological scores and noninvasive evaluation of liver fibrosis, especially after disease treatment. ${ }^{6-8}$ Nearly all morphometric studies reported so far have measured the whole fibrosis surface area in the liver specimen, regardless of its portal or lobular distribution. The first objective of our study was to develop an automated image analysis system to quantify not only the area of whole fibrosis, but also, in a separate and reliable manner, the areas of portal-bridging or perisinusoidal fibrosis on liver biopsy specimens from a large series of chronic hepatitis C. In addition, we measured the fractal dimension of whole fibrosis, portal-bridging fibrosis, and perisinusoidal fibrosis in order to explore their respective geometric organization in space. As previously shown,${ }^{9}$ fractal analysis is a mathematical method providing a quantitative characterization of complex structures that interpolate between regular geometric structures such as surfaces, lines, or points in space.

Our secondary objectives were to evaluate the respective relationships between the different fibrosis areas and the Metavir fibrosis stage and activity grade, the digitally assessed steatosis area, and, finally, the liver stiffness measurement by elastography.

Our results suggest that (1) the measure of the portal-bridging fibrosis area is a more accurate tool to assess liver fibrosis progression in chronic hepatitis $\mathrm{C}$ than the usual measure of the whole fibrosis area, and (2) the perisinusoidal fibrosis, although progressively increasing and predominant in low stages, does not influence liver stiffness evaluation.

\section{Materials and methods}

\section{Patients}

A total of 755 chronically HCV-infected patients were prospectively enrolled from March 2004 to September 2008 in three French academic centers (Angers, Bordeaux, Grenoble) or in the multicenter FIBROSTAR study promoted by the French National Agency for Research in AIDS and hepatitis. ${ }^{10}$ Patients were included if they tested positive for anti-HCV antibodies and HCV RNA in serum.
Exclusion criteria were additional causes of liver disease (eg, human immunodeficiency virus or hepatitis B virus coinfection, hemochromatosis, autoimmune hepatitis, alcohol consumption > $>30 \mathrm{~g} /$ day in men or $>20 \mathrm{~g} /$ day in women), cirrhosis complications (eg, ascites, variceal bleeding, systemic infection, and hepatocellular carcinoma), immunosuppressive treatment, or antifibrotic treatment in the past 6 months. Informed consent was obtained from each patient. The study protocol complied with the ethical guidelines of the current Declaration of Helsinki and received approval from the local Ethics committee.

\section{Liver Stiffness Measurement}

Fibroscan examination (Echosens, Paris, France) was performed using the standard probe (M probe) 1 week before liver biopsy. The manufacturer's instructions were followed. ${ }^{11}$ The experienced observer ( $>50$ Fibroscan examinations before the study) was blinded to the patient data. Fibroscan examination was discontinued after 10 valid measurements were recorded. Fibroscan results (kilopascals $(\mathrm{kPa})$ ) were expressed as the median and the interquartile range of all valid measurements.

\section{Histological Study}

Percutaneous liver biopsy was performed using a 1.4-1.6-mm-diameter needle. Specimen length was measured before paraffin-embedding. Then, $5 \mu \mathrm{m}$ thick sections of formalin-fixed, paraffin-embedded tissue were stained with hematoxylin-eosin-saffron or $0.1 \%$ picrosirius red stain and used for both optical and image analyses. Liver fibrosis and inflammatory activity were assessed according to the Metavir histological score ${ }^{2}$ by two senior experts in Angers, one senior expert in Bordeaux and Grenoble, and two senior experts in the FIBROSTAR study (central reading); in case of discordance, a consensus was reached by the experts.

\section{Image Analysis}

Image acquisition. For each patient, a picrosirius red-stained section of the whole liver biopsy was scanned using the Aperio Scanscope ${ }^{\circledR}$ CS System (Aperio Technologies, Vista, CA, USA). The image processor provided high-quality images of $30000 \times 30000$ pixels and a resolution of $0.5 \mu \mathrm{m} /$ pixel (Figure 1a). The operator manually suppressed artifacts (such as folds or dust) or liver capsule (if present) on digital images and measured the digital liver specimen's length. A binary image (black and white) of the whole specimen was obtained by automatic thresholding of the red pixels using an algorithm developed in our laboratory (Figure 1b). 


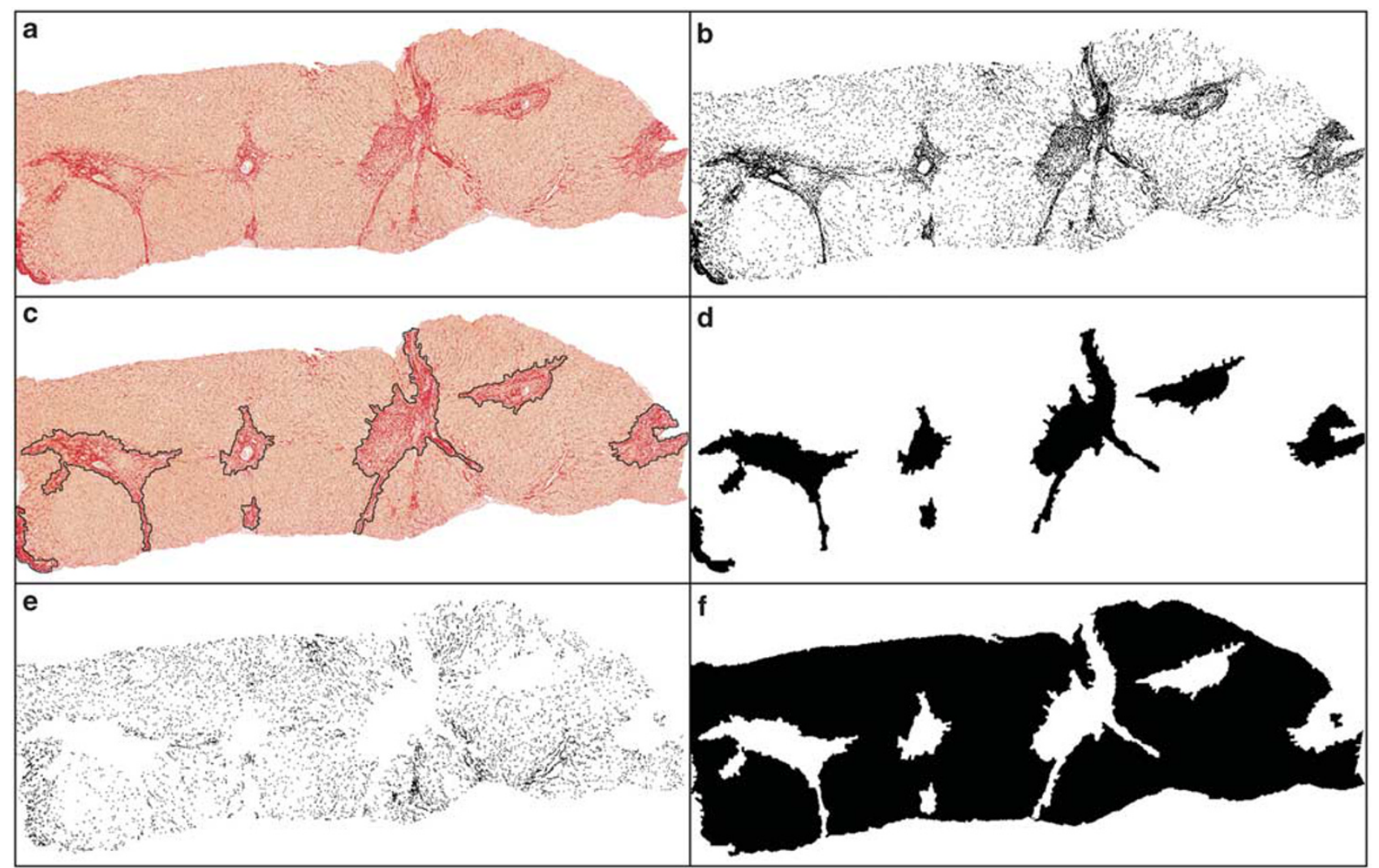

Figure 1 The process of automated image analysis. One fragment in a liver biopsy specimen of Metavir F2 stage is shown for example. (a) Digitized picrosirius red-stained section. (b) Binary image after automated threshold: fibrosis appears in black. (c) The portal and bridging fibrosis is delineated by automated segmentation. (d) The algorithm generates a mask on the portal and bridging fibrosis. (f) Binary image of perisinusoidal fibrosis without the portal and bridging fibrosis. (e) Lobular area in the whole fragment.

\section{Calculated data}

Areas of Fibrosis. The area of whole fibrosis was measured on the binary image with the use of ImageJ software. ${ }^{12}$ We developed an automated segmentation algorithm in order to separately detect and quantify the portal and/or bridging fibrosis (referred as the area of portal-bridging fibrosis), therefore bypassing the selection of areas of interest by a pathologist (Figures 1c and d). The area of perisinusoidal fibrosis relative to the lobular area was calculated by the software from all the pixels in perisinusoidal fibrosis (named PixsF, Figure 1e) and the entire lobular area (named PixLa, Figure 1f), according to the formula: area of perisinusoidal fibrosis $=$ PixsF $/$ PixLa $\times 100$. Any fibrous septum lacking liver cell plates between collagen fibers was included in the area of portalbridging fibrosis. The walls of the centrolobular veins whose caliber was $<200 \mu \mathrm{m}$ were automatically included in the area of perisinusoidal fibrosis in order not to miss small expansions of perisinusoidal fibrosis abutting veinular walls (Figure 2), whereas larger centrolobular veins were not included in that area. The automated quantification was validated by successive steps that compared, qualitatively and quantitatively, an interactive portal and bridging fibrosis selection with the automated segmentation. The final study was conducted after an excellent agreement was reached between the automated measures and the interactive procedure in a first set of 214 liver specimens (intraclass correlation coefficient for the area of portal-bridging fibrosis $=0.998$; intraclass correlation coefficient for the area of perisinusoidal fibrosis $=0.9977$ ). This validation procedure is detailed in the Supplementary Information section.

Area of Steatosis. Liver steatosis was automatically detected by the morphometry software as previously described. ${ }^{13}$ The area of steatosis corresponded to the ratio area of steatosis vacuoles/area of the whole biopsy sample.

Fractal Dimension. The box counting method provided the Kolmogorov fractal dimension for fibrosis, as already described. ${ }^{9}$ Briefly, graphs of the box counting study were performed for boxes of different sizes in order to check the slopes of regression lines and extract a fractal dimension as a characteristic of a fractal organization of liver fibrosis (detailed data not shown). Thus, calculation of the fractal dimension was separately performed for whole fibrosis, portal-bridging fibrosis, and perisinusoidal fibrosis. 


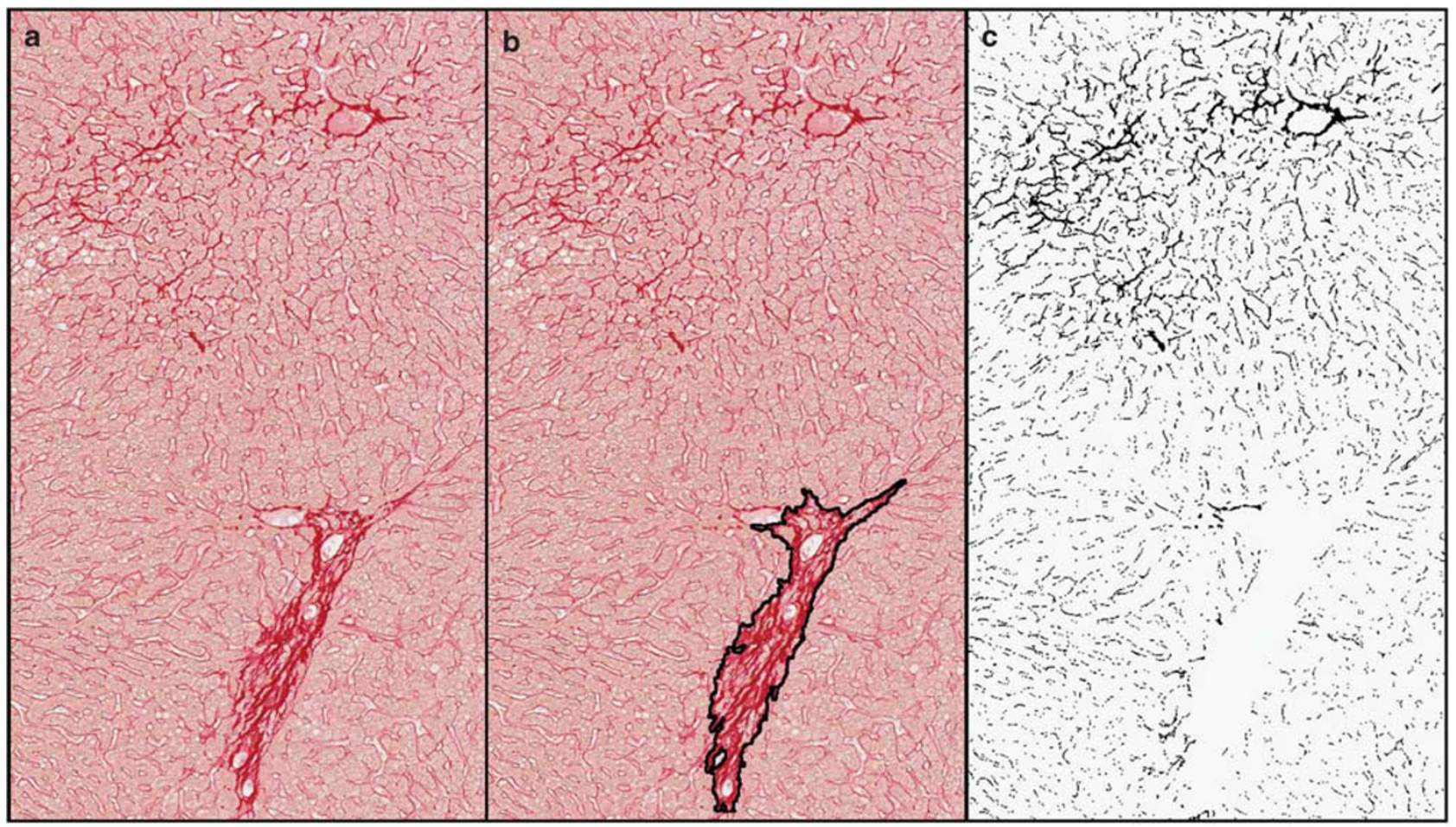

Figure 2 Perisinusoidal fibrosis in F1 stage. (a) Digitized picrosirius red-stained section showing perisinusoidal fibrosis around a centrolobular vein and a portal space at the bottom. (b) A portal area is delineated by automated segmentation. (c) Binary image of perisinusoidal fibrosis without the portal fibrosis area.

Table 1 Characteristics of the 427 patients and liver biopsies included

\section{Age (years)}

Male sex (\%)

Digitized liver biopsy length (mm)

Metavir stage F0/1/2/3/4 (\%)

Metavir grade A0/1/2/3 (\%)

$51.5 \pm 11.4$
60.3
$28 \pm 7$
$4.2 / 40.3 / 25.8 / 14.3 / 15.5$
$1.2 / 41.0 / 47.1 / 10.8$

\section{Statistical Analyses}

Quantitative variables were expressed as mean \pm s.d., unless otherwise specified. Correlations between quantitative variables were evaluated using Spearman's $(R s)$ correlation coefficient. The Lowess curve by weighted least squares was used to determine the average trend (50\% of patients) of relationships between variables. ${ }^{14}$ The statistical software programs used were SPSS version 11.5.1 (SPSS, Chicago, IL, USA) and SAS 9.1 (SAS Institute, Cary, NC, USA).

\section{Results}

\section{General Characteristics of Patients and Liver Lesions}

We excluded $28+1$ noncirrhotic patients from the initial series of 755 patients because their digitized liver specimen was $<20 \mathrm{~mm}$ in length. Also, 47 additional patients without valuable morphometric measure due to artifacts were excluded. Cirrhotic cases (Metavir F4) were included irrespective of

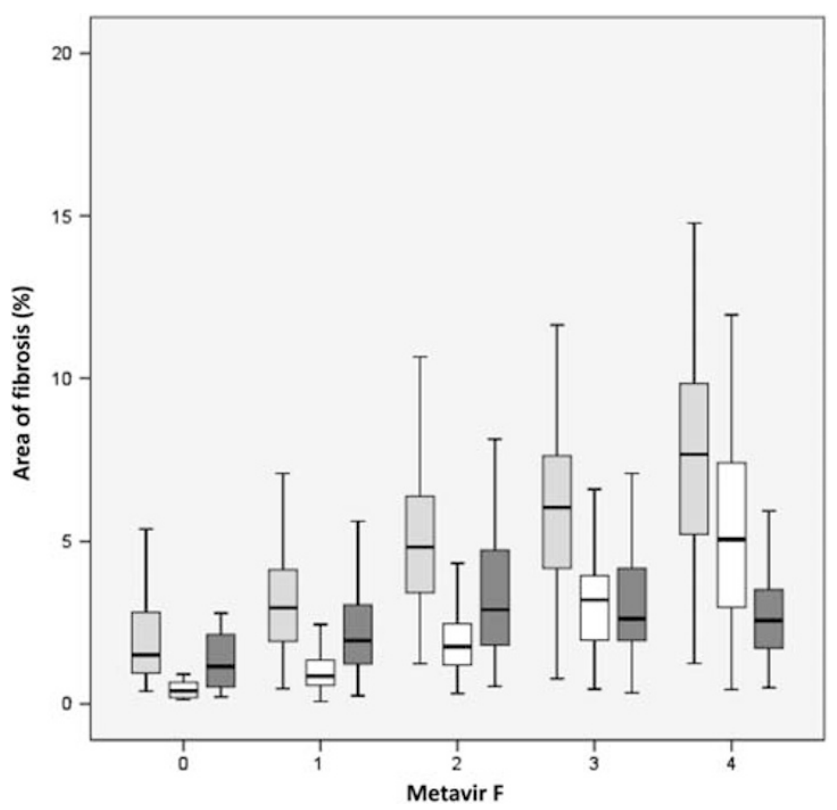

Figure 3 Distribution of fibrosis area as a function of Metavir F stage. Whole fibrosis area is in gray boxes, portal-bridging fibrosis area in white boxes, and perisinusoidal fibrosis area in dark boxes. The dark line in the middle of the box is the median, the bottom and the top of the box indicate, respectively, the 25th and 75th percentile, and the T-bars extend to 1.5 times the height of the box.

specimen length. Finally, 427 patients were included in the study. Patient and liver biopsy characteristics are detailed in Table 1 . The mean 
Table 2 Fibrosis and steatosis areas as a function of Metavir F stage

\begin{tabular}{|c|c|c|c|c|c|c|c|}
\hline \multirow[b]{2}{*}{ Areas } & \multicolumn{7}{|c|}{ Metavir F stage } \\
\hline & All & 0 & 1 & 2 & 3 & 4 & $\mathrm{P}$-value $\mathrm{a}^{\mathrm{a}}$ \\
\hline Whole fibrosis & $4.91 \pm 3.50$ & $1.89 \pm 1.36$ & $3.32 \pm 1.89$ & $5.19 \pm 2.39$ & $6.04 \pm 2.53$ & $8.39 \pm 5.64$ & $<0.001$ \\
\hline Portal-bridging fibrosis & $2.31 \pm 2.86$ & $0.62 \pm 0.76$ & $1.01 \pm 0.61$ & $1.94 \pm 1.04$ & $3.16 \pm 1.54$ & $6.00 \pm 5.33$ & $<0.001$ \\
\hline Perisinusoidal fibrosis & $2.78 \pm 1.80$ & $1.30 \pm 0.82$ & $2.38 \pm 1.63$ & $3.42 \pm 1.96$ & $3.13 \pm 1.80$ & $2.88 \pm 1.72$ & $<0.001$ \\
\hline$P$-value ${ }^{\mathrm{b}}$ & $<0.001$ & 0.002 & $<0.001$ & $<0.001$ & 0.768 & $<0.001$ & - \\
\hline $\begin{array}{l}\text { Ratio of portal-bridging/ } \\
\text { perisinusoidal fibrosis }\end{array}$ & $1.01 \pm 1.10$ & $0.49 \pm 0.35$ & $0.57 \pm 0.47$ & $0.73 \pm 0.55$ & $1.33 \pm 0.86$ & $2.45 \pm 1.78$ & $<0.001$ \\
\hline Steatosis & $2.93 \pm 3.23$ & $1.80 \pm 1.53$ & $2.44 \pm 2.62$ & $2.84 \pm 3.32$ & $3.83 \pm 4.43$ & $3.83 \pm 3.25$ & 0.001 \\
\hline
\end{tabular}

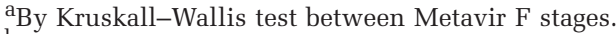

${ }^{b}$ By Wilcoxon test between the areas of portal-bridging fibrosis and perisinusoidal fibrosis.

Table 3 Pair-wise comparison of fibrosis areas between contiguous Metavir F stages ( $P$ by Mann-Whitney test)

\begin{tabular}{|c|c|c|c|c|}
\hline \multirow[b]{2}{*}{ Areas } & \multicolumn{4}{|c|}{ Metavir F stages } \\
\hline & $\begin{array}{l}F 0 \text { vs } \\
\text { F1 }\end{array}$ & $\begin{array}{c}F 1 \text { vs } \\
F 2\end{array}$ & $\begin{array}{l}F 2 \text { vs } \\
\text { F3 }\end{array}$ & $\begin{array}{c}F 3 \text { vs } \\
\text { F4 }\end{array}$ \\
\hline Whole fibrosis & $<0.001$ & $<0.001$ & 0.021 & 0.003 \\
\hline Portal-bridging fibrosis & $<0.001$ & $<0.001$ & $<0.001$ & $<0.001$ \\
\hline Perisinusoidal fibrosis & 0.002 & $<0.001$ & 0.436 & 0.429 \\
\hline $\begin{array}{l}\text { Ratio of portal-bridging/ } \\
\text { perisinusoidal fibrosis }\end{array}$ & 0.467 & 0.001 & $<0.001$ & $<0.001$ \\
\hline
\end{tabular}

length of the digitized liver biopsy was $27.6 \pm$ $7.3 \mathrm{~mm}$ in the whole cohort, and was $21.9 \pm 9.6 \mathrm{~mm}$ in the cirrhotic group.

\section{Relationships Between Quantification and Histological Scores}

Fibrosis areas as a function of fibrosis stage. As expected, the area of whole fibrosis increased as a function of Metavir F stages and was significantly different between contiguous fibrosis stages (Figure 3 and Tables 2 and 3 ). The area of perisinusoidal fibrosis increased from F0 to F2, and then remained fairly stable in F3 and F4 stages. The area of portal-bridging fibrosis showed a similar course than that of whole fibrosis. Notably, the area of portal-bridging fibrosis was significantly lower than the area of perisinusoidal fibrosis in $\mathrm{F} 0-2$ stages, was similar to the area of perisinusoidal fibrosis in F3 stage, and was markedly increased in cirrhotic patients, being significantly higher than the area of perisinusoidal fibrosis. Thus, the area of portal-bridging fibrosis represented the largest fibrotic compartment in end-stage liver disease. In addition, the area of portal-bridging fibrosis showed highly significantly different values between contiguous stages and thus better discriminated fibrosis stages than did the area of whole fibrosis.

Fractal dimension of fibrosis as a function of fibrosis stage. The distribution of the fractal dimension in

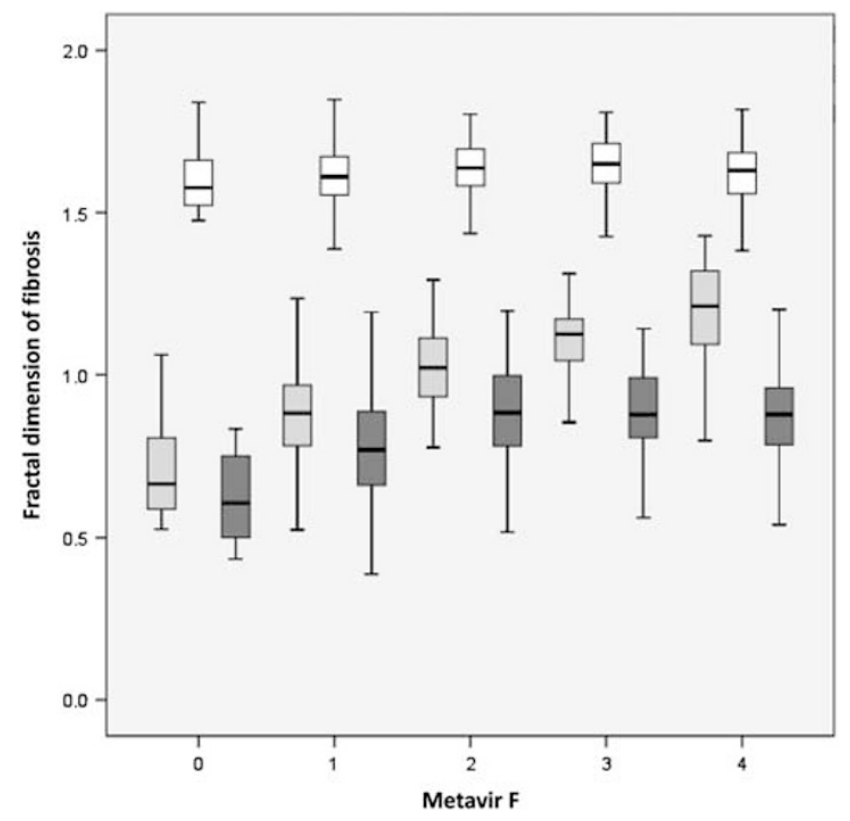

Figure 4 Distribution of fractal dimension of fibrosis as a function of Metavir $\mathrm{F}$ stage (similar signification of the box plots as in Figure 3). Whole fibrosis is in gray boxes, portal-bridging fibrosis in white boxes, and perisinusoidal fibrosis in dark boxes.

the different fibrosis compartments is shown in Figure 4. The fractal dimension of whole fibrosis showed a roughly linear increase from F0 to F4 with a significant difference between contiguous fibrosis stages (detailed data not shown). The fractal dimension of perisinusoidal fibrosis increased from F0 to F2 and then stabilized, whereas the fractal dimension of portal-bridging fibrosis was not significantly different between fibrosis stages. Whatever the Metavir F stage, the mean and median values of fractal dimension of portal-bridging fibrosis were higher than those of perisinusoidal fibrosis. The mean fractal dimension of portal-bridging fibrosis was $1.62 \pm 0.09$ that suggests, according to fractal geometry, that portal-bridging fibrosis tends to display a homogeneous surface-like organization. On the contrary, the fractal dimension of perisinusoidal fibrosis showed values closer to 1 (mean $0.83 \pm 0.17$ ) that suggests a curvilinear organization. 
Fibrosis areas as a function of hepatitis activity. The areas of whole fibrosis and of portal-bridging fibrosis significantly increased as a function of Metavir A grade (Table 4) with significant differences between contiguous grades, except between A0 and A1 for the whole fibrosis (detailed data not

Table 4 Fibrosis areas as a function of Metavir A grade

\begin{tabular}{|c|c|c|c|c|}
\hline \multirow[b]{2}{*}{ Areas } & \multicolumn{4}{|c|}{ Metavir F stages } \\
\hline & $\begin{array}{l}F 0 \text { vs } \\
\text { F1 }\end{array}$ & $\begin{array}{l}F 1 \text { vs } \\
\text { F2 }\end{array}$ & $\begin{array}{l}\text { F2 vs } \\
\text { F3 }\end{array}$ & $\begin{array}{l}\text { F3 vs } \\
\text { F4 }\end{array}$ \\
\hline Whole fibrosis & $<0.001$ & $<0.001$ & 0.021 & 0.003 \\
\hline Portal-bridging fibrosis & $<0.001$ & $<0.001$ & $<0.001$ & $<0.001$ \\
\hline Perisinusoidal fibrosis & 0.002 & $<0.001$ & 0.436 & 0.429 \\
\hline $\begin{array}{l}\text { Ratio of portal-bridging/ } \\
\text { perisinusoidal fibrosis }\end{array}$ & 0.467 & 0.001 & $<0.001$ & $<0.001$ \\
\hline
\end{tabular}

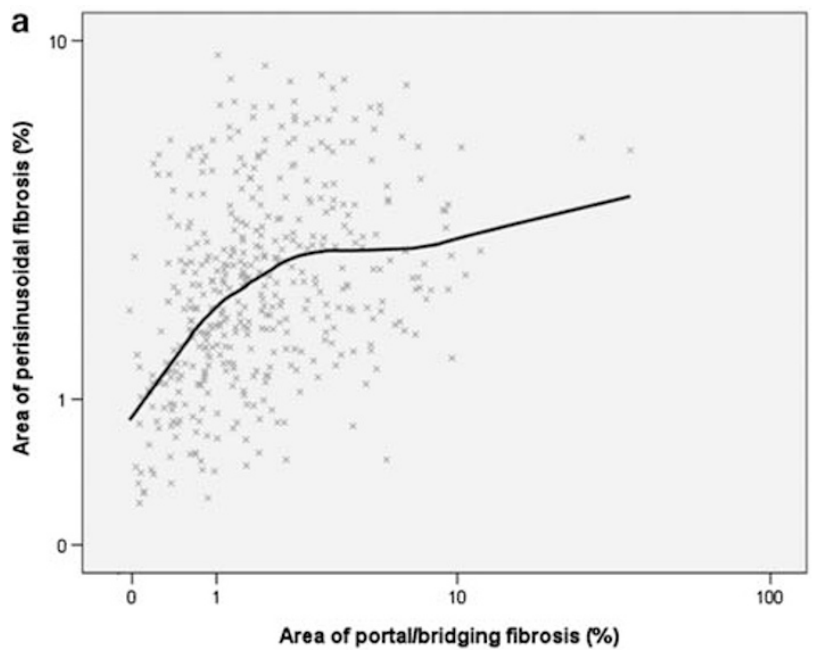

shown). The area of perisinusoidal fibrosis was significantly different between A2 and A3 grades $(P<0.001)$, but not between A0 and A1 grades, or A1 and $\mathrm{A} 2$ grades.

Steatosis area as a function of fibrosis stage. Steatosis area significantly increased as a function of Metavir F stage (Table 2). The areas of portalbridging fibrosis and of perisinusoidal fibrosis were each weakly correlated with the area of steatosis: respective Spearman's coefficient: $0.131(P=0.007)$ and $-0.144(P=0.003)$.

\section{Factors Influencing the Balance Between Portal and Perisinusoidal Fibrosis}

Figure 5a shows the average trend (Lowess curve by weighted least squares) of the relationship between the area of portal-bridging fibrosis and the area of

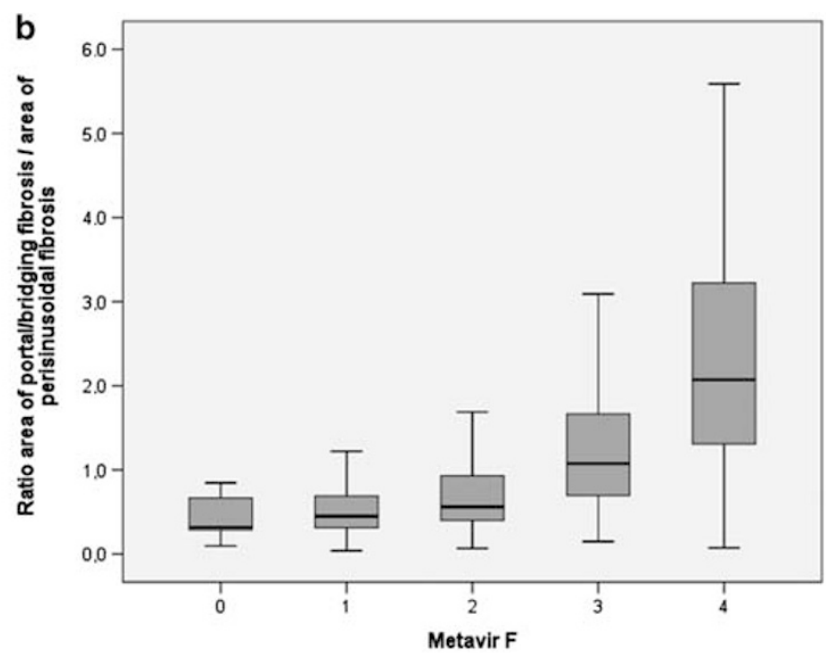

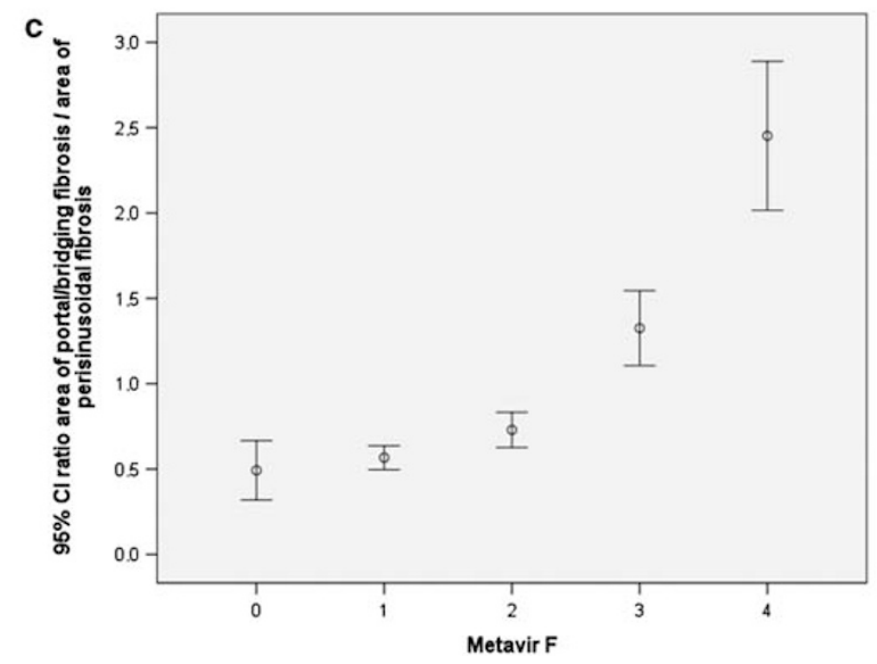

Figure 5 Relationships between the area of portal-bridging fibrosis and the area of perisinusoidal fibrosis. (a) Both areas initially increased in the same way (with the area of portal-bridging fibrosis lower than the area of perisinusoidal fibrosis), and then the area of perisinusoidal fibrosis remained relatively stable, whereas the area of portal-bridging fibrosis continued to increase. (b, c) Ratio area of portal-bridging fibrosis/area of perisinusoidal fibrosis as a function of Metavir F stage (b: box plots; c: 95\% confidence interval of the mean ratio). 
Metavir A01 and steatosis area $\leq 1.5 \%$

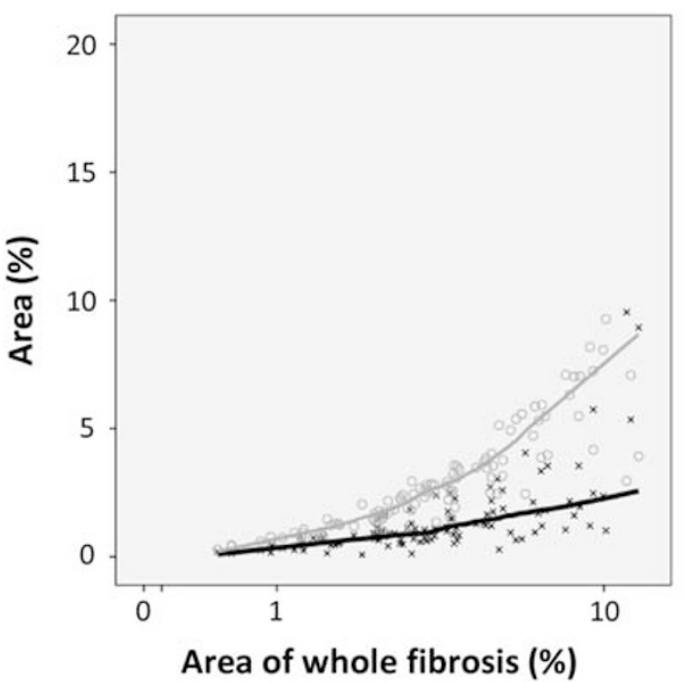

Metavir A23 and steatosis area $\leq 1.5 \%$

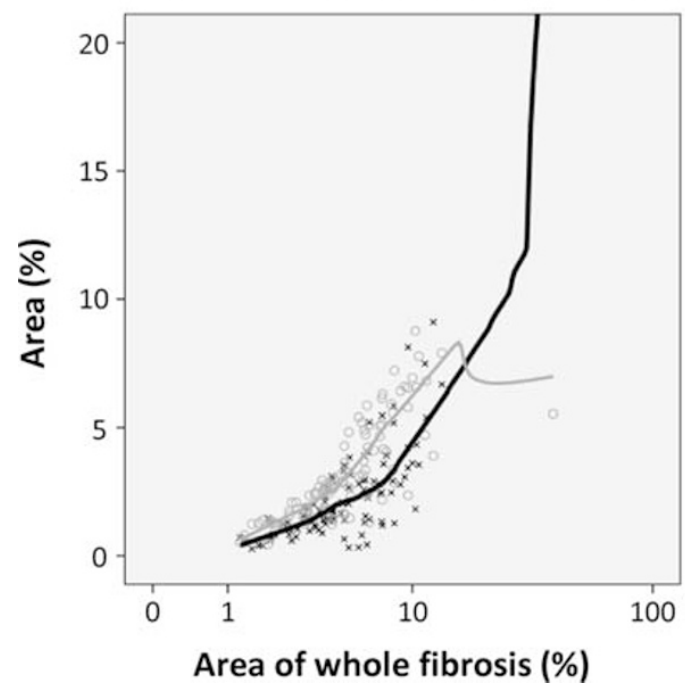

Metavir A01 and steatosis area $>1.5 \%$

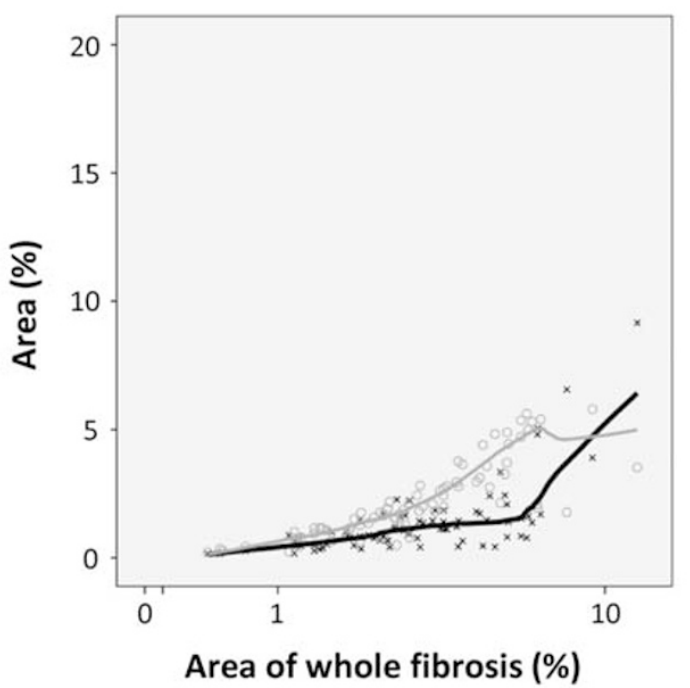

Metavir A23 and steatosis area $>1.5 \%$

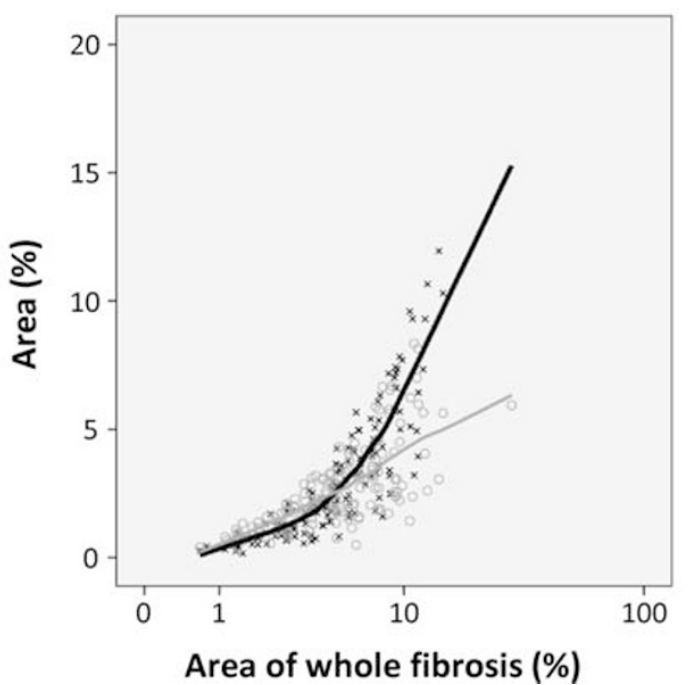

Figure 6 Contribution of the area of portal-bridging fibrosis (black slope) and of the area of perisinusoidal fibrosis (gray slope) to liver fibrosis as a function of subgroups defined by the activity and steatosis level.

perisinusoidal fibrosis. Both areas initially increased in the same way; then, the area of perisinusoidal fibrosis remained stable whereas the area of portalbridging fibrosis continued to increase. The ratio (area of portal-bridging fibrosis/area of perisinusoidal fibrosis) increased as a function of Metavir $F$ stages (Table 2) and was significantly different between contiguous fibrosis stages (except between F0 and F1, Table 3). Interestingly, as shown in Figure 5b, the boxes of this ratio showed less overlap than observed for the area of whole fibrosis in Figure 3. In addition, the ratio (area of portal-bridging fibrosis/area of perisinusoidal fibrosis) showed a remarkable gap between F2 and F3 stages (Figure 5c).

Using backward stepwise multivariate linear regression including age, sex, steatosis area, and
Metavir A (A0-1 vs A2-3) as dependent variables, the ratio (area of portal-bridging fibrosis/area of perisinusoidal fibrosis) was independently associated with Metavir A grade and steatosis area. Figure 6 shows the distribution of the area of portalbridging fibrosis and the area of perisinusoidal fibrosis in the four subgroups of patients defined by Metavir A0-1 or A2-3 and steatosis area $\leq 1.5 \%$ or $>1.5 \%$. The contribution of the area of perisinusoidal fibrosis to liver fibrosis showed a rather similar course regardless of the activity or the steatosis amount, whereas the area of portal-bridging fibrosis was low in patients with low activity and little steatosis, and became predominant with increasing activity and steatosis. 


\section{What Type of Fibrosis Influences Liver Stiffness?}

Liver stiffness measurement by Fibroscan was available for 391 out of the 427 patients included in the study. The area of portal-bridging fibrosis better correlated with the liver stiffness (expressed as LnKpa; $R s=0.583, P<0.001$ ) than did the area of whole fibrosis $(R s=0.448, P<0.001)$, the area of perisinusoidal fibrosis $(R s=0.180, P<0.001)$, the ratio (area of portal-bridging fibrosis/area of perisinusoidal fibrosis) $(R s=0.481, P<0.001)$, the area of steatosis $(R s=0.290, P<0.001)$, or the Metavir A grade $(R s=0.368, P<0.001)$. By stepwise multiple linear regression including age, sex, the area of whole fibrosis, the area of portal-bridging fibrosis, the area of perisinusoidal fibrosis, the area of steatosis, the Metavir A grade (0/1 vs 2 vs 3), the ratio of Fibroscan interquartile range/median, and the digitized biopsy length as dependent variables, the Fibroscan result (LnKpa) was independently associated to the area of portal-bridging fibrosis (first step, $P<0.001$ ), the area of steatosis (second step, $P<0.001$ ), the Metavir A grade (third step, $P=0.001$ ), the ratio of liver stiffness interquartile range/median, reflecting its reliability (fourth step, $P=0.003$ ), and the digitized biopsy length (fifth step, $P=0.010)$.

\section{Discussion}

Many previous quantifications of liver fibrosis in chronic HCV infection with image analysis found that the fibrosis ratio (ie, the proportion of the whole tissue area occupied by collagen stained by trichrome or picrosirius stain) increased with the histological stages. ${ }^{15-24}$ Notably, all these aforementioned studies used an interactive threshold to select fibrosis-related pixels that is time consuming for the operator and a possible source of intra- and interobserver variability. Moreover, none of these studies sought to determine the fibrosis ratio according to its topography in the liver tissue. In our study, we developed an original and reliable image analysis system based on an automated threshold and automated segmentation allowing a distinct quantification of whole fibrosis, portal-bridging fibrosis, and perisinusoidal fibrosis in the entire biopsy specimen. Our method was designed with picrosirius red stain and not with trichrome stain because of insufficient contrast between brown hepatocytes and green/blue collagen in the black and white binary image. We found a similar range of whole fibrosis area values in the F0, F1, and F2 stages than in a previous study using the Metavir score, ${ }^{18}$ whereas the whole fibrosis area values in the $\mathrm{F} 3$ and $\mathrm{F} 4$ stages in our series were notably lower (mean \% in the present study: $\mathrm{F} 3=6.04 ; \quad \mathrm{F} 4=8.39$ vs respectively $14.7 \%$ and $25.1 \%{ }^{18}$ ). This may be explained by distinct populations of patients with different chronic hepatitis C severity, as that study used 17 samples obtained from liver surgery for liver tumors, ${ }^{18}$ whereas we used trans-costal biopsy, mostly performed at initial evaluation of the chronic liver disease. In addition, the area of portal-bridging fibrosis not only increased as a function of the Metavir stage, as did the area of whole fibrosis, but also showed more significant different values when contiguous stages were compared.

A few morphometric studies have focused on the different fibrosis compartments in $\mathrm{CHC},{ }^{24-28}$ but owing to variations in methods, patient populations, and study goals, the results are hardly comparable to ours and between each other. The largest series in nontreated patients with chronic viral hepatitis showed that perisinusoidal fibrosis was twice more prominent in chronic hepatitis $\mathrm{C}$ than in chronic hepatitis $\mathrm{B}$ in early fibrosis stages $(\mathrm{F} \leq 2){ }^{28}$ This is an interesting finding as, unlike fibrosis in alcoholic or nonalcoholic steatohepatitis, perisinusoidal fibrosis has not been classically described as a significant lesion in CHC and is not included in the most used histological scores. In addition, a good correlation was observed between perisinusoidal fibrosis and stellate cell activation highlighted by $\alpha$-smooth muscle actin immunostaining. ${ }^{28}$ Stellate cell activation in CHC is likely, as in vitro interactions have been described between these cells and HCV components ${ }^{29,30}$ and results in progressive deposition of type I and III collagen fibrils that are easily identified with the picrosirius stain. ${ }^{7}$ In our study, we defined perisinusoidal fibrosis as picrosiriusstained fibrils separated by liver cell plate. Fibrous septa resulting from the thick coalescence of perisinusoidal fibrosis were excluded from the quantification of perisinusoidal fibrosis. With this strict definition, we showed that in early fibrosis stages $(F \leq 2)$, there was a significant increase of perisinusoidal fibrosis from F0 to F2, and that mean and median values of the area of perisinusoidal fibrosis were higher than those of portal-bridging fibrosis. In subsequent stages F3 and F4, the area of perisinusoidal fibrosis stabilized that may be explained by the growing proportion of liver tissue occupied by thickened septa and cirrhotic annular fibrosis along with loss of hepatocytes. A caveat in the interpretation of morphometric results is the lack of normal range values for fibrosis area in healthy adult livers. As F0 is defined as the absence of portal fibrosis, one may logically assume that the very low mean and median area of portal fibrosis values in F0 reflect the normal portal collagen content. The proportion of normal vs pathological perisinusoidal fibrosis area remains unknown in F0. Nonetheless, our study clearly showed that a detectable and pathologic perisinusoidal collagen network is present in early fibrosis stages (F0 to F2) in CHC, with a quite wide range of values, as previously observed. ${ }^{28}$

The mere quantification of fibrosis areas cannot describe the spatial organization of liver fibrosis. We thus provided by fractal analysis a quantitative index, the fractal dimension, that reflects the 
geometry of complex structures in space. Regular structures usually display integer dimensions: a solid surface has a dimension of 2, whereas a curvilinear structure has a dimension of 1 , and isolated points have a dimension of 0 . A fractal structure can show a noninteger dimension between 0 and 2, and this is usually associated with lacunarity or concentration over many spatial scales. As highlighted in Figure 4, the distribution of the fractal dimensions as a function of Metavir stage was very different between portal-bridging fibrosis and perisinusoidal fibrosis. The mean fractal dimension of portal-bridging fibrosis value suggests a surface-type expansion, whereas that of perisinusoidal fibrosis suggests a curvilinear organization. In addition, the relative stability of the fractal dimension of portal-bridging fibrosis contrasts with the regular increase of the fractal dimension of perisinusoidal fibrosis as a function of Metavir stage. Further studies will be useful to explore the complex interactions governing the spatial distribution of whole fibrosis, portalbridging fibrosis, and perisinusoidal fibrosis in order to simultaneously ensure fractal organizations for the three fibrosis compartments.

Despite numerous studies, there is no clearcut consensus about the relationships between steatosis, inflammation, and fibrosis in chronic hepatitis C. In patients infected with HCV genotype 3, steatosis seems related to a direct cytopathic effect, whereas in genotype 1, steatosis is mainly related to an associated metabolic syndrome. ${ }^{31,32}$ Clouston et $a l^{33}$ noticed a statistically significant association between subsinusoidal fibrosis and the steatosis grade (both histologically assessed without morphometry), although the latter did not correlate with the extent of $\alpha$-smooth muscle actin immunostaining as a marker of stellate cell activation. Subsequently, Walsh et al $\mathrm{l}^{34}$ observed that, in the presence of steatosis, apoptosis was associated with activation of stellate cells and increased stage of fibrosis. Moreover, patients with subsinusoidal fibrosis had a higher mean body mass index than those without subsinusoidal fibrosis, which suggested a role of the metabolic syndrome. In a meta-analysis, ${ }^{35}$ the presence of necroinflammatory activity was independently associated with the presence of fibrosis and steatosis. But in another large cohort, ${ }^{36}$ steatosis was associated with higher body mass index but not with the presence, or subsequent progression, of fibrosis. Bedossa et al $\mathrm{l}^{37}$ further demonstrated that HCV-infected patients with nonalcoholic steatohepatitis showed a higher Metavir fibrosis stage than HCV-infected patients with only steatosis, but perisinusoidal fibrosis was not mentioned in this study. In our series, the ratio (area of portal-bridging fibrosis/area of perisinusoidal fibrosis) was independently associated with the Metavir A grade and the steatosis area. However, this observation cannot explain a mechanistic link between these lesions.

Noninvasive methods to evaluate liver fibrosis have mainly been developed on the basis of histological scores as the best reference test to assess the accuracy of discriminating 'clinically significant fibrosis' and/or cirrhosis. ${ }^{4}$ Some studies also used image analysis of liver tissue to evaluate correlations between the proportion of whole collagen fibrosis and transient elastography. ${ }^{27,38-40}$ Wong et $a l^{27}$ observed in an univariate analysis that 'pericellular fibrosis' better correlated with liver stiffness measurement than 'periportal' or 'perivenular fibrosis,' especially in patients with severe fibrosis. This study and ours are barely comparable, because of size difference and different methodology. In contrast, we found that portal-bridging fibrosis correlated with liver stiffness measurement more accurately than did whole fibrosis or perisinusoidal fibrosis. Moreover, after multivariate analysis, portal-bridging fibrosis appeared as the first independent variable associated with liver stiffness measurement. Our observation suggests that misclassification of patient's stage that may occur with Fibroscan is not influenced by perisinusoidal fibrosis.

In summary, we have designed a fully automated image analysis system to separately quantify whole fibrosis, portal-bridging fibrosis, and perisinusoidal fibrosis in a large series of chronic hepatitis $C$. We have shown that Metavir stage F2, which is usually considered as the start point of 'significant fibrosis' in clinical practice, indeed appeared as a pivotal stage in the natural history of HCV fibrosis: perisinusoidal fibrosis progressively develops with an area higher than that of portal fibrosis in stages $\leq$ F2 and then stabilizes, whereas portoseptal fibrosis area accounts for the largest compartment in cirrhosis. Analysis of fractal geometry in the distinct fibrosis compartments suggested that portalbridging fibrosis and perisinusoidal fibrosis display different types of spatial organization, with complex interactions as yet to unravel. The area of portalbridging fibrosis and the ratio (area of portalbridging fibrosis/area of perisinusoidal fibrosis) more accurately discriminated fibrosis stages than the usual whole fibrosis quantification. Portal-bridging fibrosis showed various correlations with steatosis and hepatitis activity and highly correlated with liver stiffness measured by transient elastrography. Our study does not support the necessity of a routine assessment of perisinusoidal fibrosis in chronic hepatitis C. This new image analysis method may be a reliable, reproducible, and precise tool in large cohorts where noninvasive tests and histologic examination are used to evaluate liver fibrosis in its natural or posttherapy history.

\section{Acknowledgments}

This study was supported by ANRS (French national agency for AIDS and Viral Hepatitis) for HC/EP23 Fibrostar. 


\section{Disclosure/conflict of interest}

The authors declare no conflict of interest.

\section{References}

1 Ghany MG, Strader DB, Thomas DL, et al. American Association for the Study of Liver Diseases. Diagnosis, management, and treatment of hepatitis C: an update. Hepatology 2009;49:1335-1374.

2 The French METAVIR Cooperative Study Group. Intraobserver and interobserver variations in liver biopsy interpretation in patients with chronic hepatitis C. Hepatology 1994;20:15-20.

3 Ishak K, Baptista A, Bianchi L, et al. Histological grading and staging of chronic hepatitis. J Hepatol 1995;22:696-699.

4 Stauber RE, Lackner C. Noninvasive diagnosis of hepatic fibrosis in chronic hepatitis C. World J Gastroenterol 2007;13:4287-4294.

5 Standish RA. An appraisal of the histopathological assessment of liver fibrosis. Gut 2006;55:569-578.

6 Germani G, Burroughs AK, Dhillon AP. The relationship between liver disease stage and liver fibrosis: a tangled web. Histopathology 2010;57:773-784.

7 Germani G, Hytiroglou P, Fotiadu A, et al. Assessment of fibrosis and cirrhosis in liver biopsies: an update. Semin Liver Dis 2011;31:82-90.

8 Goodman ZD, Becker RL, Pockros PJ, et al. Progression of fibrosis in advanced chronic hepatitis C: Evaluation by morphometric image analysis. Hepatology 2007;45: 886-894.

9 Moal F, Chappard D, Wang J, et al. Fractal dimension can distinguish models and pharmacologic changes in liver fibrosis in rats. Hepatology 2002;36:840-849.

10 Zarski JP, Sturm N, Guechot J, et al. Comparison of nine blood tests and transient elastography for liver fibrosis in chronic hepatitis C: the ANRS HCEP-23 study. J Hepatol 2012;56:55-62.

11 Castera L, Forns X, Alberti A. Non-invasive evaluation of liver fibrosis using transient elastography. J Hepatol 2008;48:835-847.

12 Rasband WS. Image U.S. National Institutes of Health, Bethesda, Maryland, USA, imagej.nih.gov/ij/, 1997-2011.

13 Boursier J, Chaigneau J, Roullier V, et al. Steatosis degree, measured by morphometry, is linked to other liver lesions and metabolic syndrome components in patients with NAFLD. Eur J Gastroenterol Hepatol 2011;23:974-981.

14 Borkowf CB, Albert PS, Abnet CC. Using lowess to remove systematic trends over time in predictor variables prior to logistic regression with quantile categories. Stat Med 2003;22:1477-1493.

15 Duchatelle V, Marcellin P, Giostra E, et al. Changes in liver fibrosis at the end of alpha interferon therapy and 6 to 18 months later in patients with chronic hepatitis C: quantitative assessment by a morphometric method. J Hepatol 1998;29:20-28.

16 Pilette C, Rousselet MC, Bedossa P, et al. Histopathological evaluation of liver fibrosis: quantitative image analysis vs semi-quantitative scores. Comparison with serum markers. J Hepatol 1998;28:439-446.

17 O’Brien MJ, Keating NM, Elderiny S, et al. An assessment of digital image analysis to measure fibrosis in liver biopsy specimens of patients with chronic hepatitis C. Am J Clin Pathol 2000;114:712-718.
18 Bedossa P, Dargère D, Paradis V. Sampling variability of liver fibrosis in chronic hepatitis C. Hepatology 2003;38:1449-1457.

19 Calvaruso V, Burroughs AK, Standish R, et al. Computer-assisted image analysis of liver collagen: relationship to Ishak scoring and hepatic venous pressure gradient. Hepatology 2009;49:1236-1244.

20 Fontana RJ, Goodman ZD, Dienstag JL, et al. Relationship of serum fibrosis markers with liver fibrosis stage and collagen content in patients with advanced chronic hepatitis C. Hepatology 2008;47:789-798.

21 Lazzarini AL, Levine RA, Ploutz-Snyder RJ, et al. Advances in digital quantification technique enhance discrimination between mild and advanced liver fibrosis in chronic hepatitis C. Liver Int 2005;25: 1142-1149.

22 Levine RA, Sanderson SO, Ploutz-Snyder R, et al. Assessment of fibrosis progression in untreated Irish women with chronic hepatitis $\mathrm{C}$ contracted from immunoglobulin anti-D. Clin Gastroenterol Hepatol 2006;4:1271-1277.

23 Zarski JP, Sturm N, Desmorat H, et al. Non-invasive assessment of liver fibrosis progression in hepatitis $\mathrm{C}$ patients retreated for 96 weeks with antiviral therapy: a randomized study. Liver Int 2010;30: 1049-1058.

24 Moragas A, Allende H, Sans M. Characteristics of perisinusoidal collagenization in liver cirrhosis: computer-assisted quantitative analysis. Anal Quant Cytol Histol 1998;20:169-177.

25 Masseroli M, Caballero T, O’Valle F, et al. Automatic quantification of liver fibrosis: design and validation of a new image analysis method: comparison with semi-quantitative indexes of fibrosis. J Hepatol 2000; 32:453-464.

26 Caballero T, Pérez-Milena A, Masseroli M, et al. Liver fibrosis assessment with semiquantitative indexes and image analysis quantification in sustained-responder and non-responder interferon-treated patients with chronic hepatitis C. J Hepatol 2001;34:740-747.

27 Wong GLH, Wong VWS, Choi PCL, et al. Assessment of fibrosis by transient elastography compared with liver biopsy and morphometry in chronic liver diseases. Clin Gastroenterol Hepatol 2008;6:1027-1035.

28 Sturm N, Marlu A, Arvers P, et al. Comparative assessment of liver fibrosis by computerized morphometry in naïve patients with chronic hepatitis B and C. Liver Int 2013;33:428-438.

29 Bataller R, Paik Y-H, Lindquist JN, et al. Hepatitis C virus core and nonstructural proteins induce fibrogenic effects in hepatic stellate cells. Gastroenterology 2004;126:529-540.

30 Schulze-Krebs A, Preimel D, Popov Y, et al. Hepatitis C virus-replicating hepatocytes induce fibrogenic activation of hepatic stellate cells. Gastroenterology 2005; 129:246-258.

31 Rubbia-Brandt L, Quadri R, Abid K, et al. Hepatocyte steatosis is a cytopathic effect of hepatitis $\mathrm{C}$ virus genotype 3. J Hepatol 2000;33:106-115.

32 Adinolfi LE, Gambardella $\mathrm{M}$, Andreana A, et al. Steatosis accelerates the progression of liver damage of chronic hepatitis $\mathrm{C}$ patients and correlates with specific HCV genotype and visceral obesity. Hepatology 2001;33:1358-1364.

33 Clouston AD, Jonsson JR, Purdie DM, et al. Steatosis and chronic hepatitis $\mathrm{C}$ : analysis of fibrosis and stellate cell activation. J Hepatol 2001;34:314-320. 
34 Walsh MJ, Vanags DM, Clouston AD, et al. Steatosis and liver cell apoptosis in chronic hepatitis C: a mechanism for increased liver injury. Hepatology 2004;39:1230-1238.

35 Leandro G, Mangia A, Hui J, et al. Relationship between steatosis, inflammation, and fibrosis in chronic hepatitis C: a meta-analysis of individual patient data. Gastroenterology 2006;130:1636-1642.

36 Perumalswami $\mathrm{P}$, Kleiner DE, Lutchman G, et al. Steatosis and progression of fibrosis in untreated patients with chronic hepatitis $\mathrm{C}$ infection. Hepatology 2006;43:780-787.

37 Bedossa P, Moucari R, Chelbi E, et al. Evidence for a role of nonalcoholic steatohepatitis in hepatitis C: a prospective study. Hepatology 2007;46:380-387.
38 Ziol M, Kettaneh A, Ganne-Carrié N, et al. Relationships between fibrosis amounts assessed by morphometry and liver stiffness measurements in chronic hepatitis or steatohepatitis. Eur J Gastroenterol Hepatol 2009;21:1261-1268.

39 Nitta Y, Kawabe N, Hashimoto S, et al. Liver stiffness measured by transient elastography correlates with fibrosis area in liver biopsy in patients with chronic hepatitis C. Hepatol Res 2009;39:675-684.

40 Boursier J, de Ledinghen V, Sturm N, et al. Precise evaluation of liver histology by computerized morphometry shows that steatosis influences liver stiffness measured by transient elastography in chronic hepatitis C. J Gastroenterol 2013; doi:10. 1007/s00535-013-0819-9.

Supplementary Information accompanies the paper on Modern Pathology website (http://www.nature.com/ modpathol) 